\section{Optimized EBMT transplant-specific risk score in myelodysplastic syndromes after allogeneic stem-cell transplantation}

\author{
Nico Gagelmann, ${ }^{1}$ Diderik-Jan Eikema, ${ }^{2}$ Matthias Stelljes, ${ }^{3}$ Dietrich Beelen, ${ }^{4}$ \\ Liesbeth de Wreede, ${ }^{2}$ Ghulam Mufti ${ }^{5}$, Nina Simone Knelange, ${ }^{6}$ Dietger \\ Niederwieser, ${ }^{7}$ Lone S. Friis, ${ }^{8}$ Gerhard Ehninger, ${ }^{9}$ Arnon Nagler, ${ }^{10}$ Ibrahim \\ Yakoub-Agha, ${ }^{11}$ Ellen Meijer, ${ }^{12}$ Per Ljungman, ${ }^{13}$ Johan Maertens, ${ }^{14}$ Lothar \\ Kanz,${ }^{15}$ Lucia Lopez-Corral, ${ }^{16}$ Arne Brecht, ${ }^{17}$ Charles Craddock, ${ }^{18}$ Jürgen Finke, ${ }^{19}$ \\ Jan J. Cornelissen, ${ }^{20}$ Paolo Bernasconi, ${ }^{21}$ Patrice Chevallier, ${ }^{22}$ Jorge Sierra, ${ }^{23}$ \\ Marie Robin ${ }^{24}$ and Nicolaus Kröger ${ }^{1}$
}

${ }^{1}$ University Medical Center Hamburg-Eppendorf, Hamburg, Germany; ${ }^{2}$ EBMT Statistics, Leiden, the Netherlands; ${ }^{3}$ University of Münster, Germany; ${ }^{4}$ Department of Bone Marrow Transplantation, West German Cancer Center, University Hospital of Essen, Germany; ${ }^{5}$ GKT School of Medicine, London, UK; ${ }^{6}$ EBMT Data Office, Leiden, the Netherlands; ${ }^{7}$ University Hospital Leipzig, Germany; ${ }^{8}$ Rigshospitalet, Copenhagen, Denmark; ${ }^{9}$ Universitätsklinikum Dresden, Germany; ${ }^{10} \mathrm{Chaim}$ Sheba Medical Center, Tel-Hashomer, Israel; ${ }^{11} \mathrm{CHU}$ de Lille, LIRIC, INSERM U995, Université Lille2, France; ${ }^{12} \mathrm{VU}$ University Medical Center, Amsterdam, the Netherlands; ${ }^{13}$ Karolinska University Hospital and Karolinska Institutet, Stockholm, Sweden; ${ }^{14}$ University Hospital Gasthuisberg, Leuven, Belgium; ${ }^{15}$ Universität Tübingen, Germany; ${ }^{16} \mathrm{Hospital}$ Clínico, Salamanca, Spain; ${ }^{17}$ Deutsche Klinik für Diagnostik, Wiesbaden, Germany; ${ }^{18}$ Centre for Clinical Haematology, Birmingham, UK; ${ }^{19}$ University of Freiburg, Germany; ${ }^{20}$ Erasmus MC Cancer Institute, Erasmus University Medical Center, Rotterdam, the Netherlands; ${ }^{21}$ Fondazione IRCCS Policlinico San Matteo, Pavia, Italy; ${ }^{22} \mathrm{CHU}$ Nantes, France; ${ }^{23} \mathrm{Hospital}$ Santa Creu i Sant Pau, Jose Carreras Leukemia Research Institute, Barcelona, Spain and ${ }^{24}$ Hopital St. Louis, Paris, France

\section{Ferrata Storti Foundation} 2019 Volume 104(5):929-936

\section{Correspondence:}

NICOLAUS KRÖGER

nkroeger@uke.uni-hamburg.de

T The aim of this study was to develop and validate a clinical and transplant-specific prognostic score using data from a large cohort of patients with myelodysplastic syndromes reported to the European Society for Blood and Marrow Transplantation registry. A Cox model was fitted to detect clinical and transplant-related variables prognostic of outcome. Then, cross-validation was performed to evaluate the validity and consistency of the model. Seven independent risk factors for survival were identified: age $\geq 50$ years, matched unrelated donor, Karnofsky Performance Status $<90 \%$, very poor cytogenetics or monosomal karyotype, positive cytomegalovirus status of the recipient, blood blasts $>1 \%$, and platelet count $\leq 50 \times 10^{9} / \mathrm{L}$ prior to transplantation. Incorporating these factors into a four-level risk score yielded hazard ratios for death, with low-risk (score of $0-1)$ as reference, of 2.02 (95\% CI: 1.41-2.90) for the intermediate-risk group (score of 2-3), 3.49 (95\% CI: 2.45-4.97) for the high-risk group (score of 4-5), and 5.90 (95\% CI: 4.01-8.67) for the very high-risk group (score of $>5$ ). The score was predictive of survival, relapse-free survival, relapse, and non-relapse mortality $(P<0.001$, respectively). Cross-validation yielded significant and reproducible improvement in prognostic ability with C-statistics being 0.609 (95\% CI: 0.588-0.629) versus 0.555 for the Gruppo Italiano Trapianto di Midollo Osseo registry and 0.579 for the Center for Blood and Marrow Transplant Research registry. Prediction was even further augmented after applying a nomogram using age and platelets as continuous variables showing C-statistics of 0.628 (95\% CI: 0.616-0.637). In conclusion, compared to existing prognostic systems, this proposed transplantspecific risk score offers improved performance with respect to post-transplant risk stratification in myelodysplastic syndromes.
Received: July 2, 2018.

Accepted: January 9, 2019.

Pre-published: January 17, 2019.

doi:10.3324/haematol.2018.200808

Check the online version for the most updated information on this article, online supplements, and information on authorship \& disclosures: www.haematologica.org/content/104/5/929

(C)2019 Ferrata Storti Foundation

Material published in Haematologica is covered by copyright. All rights are reserved to the Ferrata Storti Foundation. Use of published material is allowed under the following terms and conditions:

https://creativecommons.org/licenses/by-nc/4.0/legalcode. Copies of published material are allowed for personal or internal use. Sharing published material for non-commercial purposes is subject to the following conditions:

https://creativecommons. org/licenses/by-nc/4.0/legalcode, sect. 3. Reproducing and sharing published material for commercial purposes is not allowed without permission in writing from the publisher. 


\section{Introduction}

Myelodysplastic syndromes (MDS) are a heterogeneous group of clonal hematopoietic disorders that are characterized by abnormal cellular maturation resulting in cytopenia and a variable risk of progression to acute leukemia. ${ }^{1}$ Allogeneic stem-cell transplantation is still the only curative treatment option. ${ }^{2.4}$ In order to recommend MDS patients for transplantation besides considering only disease-specific factors, such as those proposed by the International Prognostic Scoring System (IPSS) and its revision (IPSS-R), ,5,6 valid and readily reproducible transplant-specific scoring system is needed. ${ }^{7,8}$

Recently, two groups have developed prognostic systems incorporating patient-specific as well as transplantspecific factors. The Gruppo Italiano Trapianto di Midollo Osseo (GITMO) registry developed a transplantation risk index consisting of the following factors: age $(<50$ or $\geq 50$ years), IPSS-R score (low, intermediate, high, or very high), monosomal karyotype (yes or no), refractoriness to chemotherapy (yes or no), and Hematopoietic Cell Transplantatiom Comorbidity Index score (HCT-CI). The resulting risk index could clearly distinguish four different groups (low, intermediate, high, and very high) with corresponding overall survival rates at 5 years of $76 \%, 48 \%, 18 \%$, and $5 \%$. The other prognostic score, by the Center for Blood and Marrow Transplant Research (CIBMTR) registry, included the following criteria: age $(18-29,30-49$, or $\geq 50$ years), Karnofsky status (90-100 or $<90 \%$ ), cytogenetics (very good to intermediate, poor, very poor or monosomal karyotype), blood blasts before transplantation $(>3$ or $\leq 3 \%$ ) and platelet count before transplantation $\left(>50\right.$ or $\leq 50 \times 10^{9} / \mathrm{L}$ ). This system also discriminated four risk groups (low, intermediate, high, and very high) and showed corresponding overall survival rates at 3 years of $71 \%, 49 \%, 41 \%$, and $25 \% .{ }^{10}$ Both systems resulted in relevant re-classification of patients in comparison with the IPSS-R while providing only modest improvement in predictability. While neither score has been externally validated nor investigated regarding prognostic power in large and independent cohorts, we hypothesized that both scores not only vary in design and follow-up but would also vary in prognostic ability when applied to the same cohort. ${ }^{7,8}$

We, therefore, aimed to develop and validate a clinical and transplant-specific prognostic score using data from a large cohort of MDS patients reported to the European Society for Blood and Marrow Transplantation (EBMT) registry and to validate and compare currently existing systems with respect to the resulting EBMT transplantspecific risk score.

\section{Methods}

\section{Data source}

The EBMT is a voluntary organization that comprises more than 500 transplant centers, mainly from Europe. Membership requires submission of the minimal essential data form $\mathrm{A}$ for all consecutive patients to a central registry from which patients can be identified by diagnosis of underlying disease and type of transplantation. The information in the minimal essential data form A data is updated annually. Informed consent to transplantation was obtained and data were collected locally according to regulations that were applicable at the time of transplantation. All transplantation centers were required to obtain written informed consent before data registration with the EBMT in accordance with the 1975 Helsinki Declaration.

\section{Patients}

Adult patients ( $\geq 18$ years) with MDS who underwent transplantation from an HLA-identical sibling or matched unrelated donor between 2000 to 2014 were included. Patients were eligible if there was full information on their: diagnosis, donor data, cytogenetic risk, platelet count and blood blasts at transplantation. Cytogenetic risk was stratified based on previously established systems. ${ }^{6,11}$ The prognostic subgroups were the following: $\operatorname{del}(11 q)$ and $-Y($ very good); $\operatorname{del}(5 q), \operatorname{del}(12 p), \operatorname{del}(20 q)$, and normal karyotype (good); del(7q), +8, i(17q), +19, and other independent clones (intermediate); complex karyotype (three abnormalities), inv(3), del(3q), and translocations involving $3 q$ (poor), and very complex karyotype with more than three abnormalities (very poor). Monosomal karyotype was defined as monosomy of two or more chromosomes or one single autosomal monosomy in the presence of other structural abnormalities. ${ }^{12}$ The IPSS-R was calculated prior to transplantation. In total, 1059 patients met the criteria and were included in the EBMT cohort. To evaluate possible selection bias, outcomes were compared between the final cohort and remaining patients not included in the analysis due to missing data in the registry $(n=5122)$. Within the EBMT cohort, 519 and 876 patients had full data on all factors included in either the GITMO or CIBMTR score.

\section{Score development}

The development of the transplant-specific risk score consisted of two steps. First, a Cox proportional hazards model using backward and forward selection was used to identify significant covariates for overall survival. ${ }^{13}$ Then, the hazard ratios (HR) obtained were classified as large (HR >1.59), intermediate $(1.25<$ $\mathrm{HR}<1.60)$ and small $(\mathrm{HR}<1.25)$. Subsequently, a scoring rule was defined in which large effects were assigned two points, intermediate effects were assigned one point and small effects were assigned zero points. Scores were grouped based on associated hazard ratios into low-, intermediate-, high-, and very high-risk groups, providing group-based risk predictions for MDS. A second score was developed, based on the $\beta$ coefficients derived from the model defined above, to provide individualized/patient-specific risk predictions. Second, both developed scores were validated and then compared to existing systems by assessing each score's prognostic performance.

\section{Statistical analysis}

Overall survival and relapse-free survival were estimated using the Kaplan-Meier method and compared with the log-rank test in univariable analysis. Non-relapse mortality and relapse were analyzed in a competing risks framework by using the cumulative incidence estimator and the Gray test for univariable analysis. ${ }^{14}$ Cox proportional hazards regression of complete data was used to develop the two new scores. Maximum likelihood from the Cox model was used to establish cutoffs for continuous variables. Score performance was analyzed using the concordance index (C): the probability that a patient who experienced an event had a higher risk score than a patient who did not ( $C>0.5$ suggesting predictive ability). ${ }^{15,16}$ Each system was validated using 5-fold cross-validation with 100 repetitions. $P$ values $<0.05$ were considered statistically significant. Analyses were performed using SPSS for Windows version 24 (SPSS, Chicago, IL, USA) and R package version 3.4.3 (The R Foundation, Vienna, Austria). 


\section{Results}

\section{Patients}

The characteristics of the patients and transplants of the total EBMT cohort $(\mathrm{n}=1059)$ are listed in Table 1 . The median follow-up was 69 months (95\% CI: 62-76) and the 5 -year overall survival was $38.6 \%$ (95\% CI: $35.4-41.7)$ while the relapse-free survival was $33.5 \%$ (95\% CI: $30.4-$ 36.6).

\section{Transplant-specific risk score}

Table 2 summarizes the seven variables identified as independent predictors of overall survival in the multivariable analysis: age $\geq 50$ years, matched unrelated donor, Karnofsky Performance Status $<90 \%$, very poor cytogenetics or monosomal karyotype, positive cytomegalovirus (CMV) status of the recipient, blood blasts $>1 \%$, and platelet count $\leq 50 \times 10^{9} / \mathrm{L}$ at the time of transplantation. A weighted score of two was assigned to older age ( $\geq 50$ years) and very poor cytogenetics or monosomal karyotype, whereas matched unrelated donor, Karnofsky Performance Status $<90 \%$, positive CMV status of the recipient, blood

Table 1. Patient and transplantation characteristics of 1059 patients with myelodysplastic syndromes in the total EBMT cohort.

\begin{tabular}{lc} 
Characteristics & Total cohort, n (\%) \\
Number of patients & 1059 \\
Age at transplant, years & \\
median (range) & $56(18$ to 73$)$ \\
\hline Patient's sex & \\
female & $446(42)$ \\
male & $613(58)$ \\
Secondary disorder & \\
no & $776(74)$ \\
yes & $201(19)$ \\
unknown & $92(7)$ \\
\hline Karnofsky index, $\%$ & \\
90 to 100 & $630(60)$ \\
$<90$ & $252(23)$ \\
unknown & $177(17)$ \\
Comorbidity index, $\%$ & \\
0 to 2 & $462(44)$ \\
$\geq 3$ & $190(18)$ \\
unknown & $407(38)$ \\
\hline Cytogenetic risk & \\
very good & $26(3)$ \\
good & $132(13)$ \\
intermediate & \\
poor & $357(34)$ \\
very poor & $299(28)$ \\
monosomal karyotype & $45(4)$ \\
Marrow blasts at transplant, \% & $200(19)$ \\
2 to 5 & \\
5 to 10 & $333(31)$ \\
$>10$ & $258(24)$ \\
unknown & $170(16)$ \\
Blood blasts at transplant, \% & $114(12)$ \\
$\leq 1$ & $210(17)$ \\
$>1$ & \\
& $929(88)$ \\
\hline & $130(12)$ \\
\hline & \\
\hline
\end{tabular}

blasts $>1 \%$, and platelet count $\leq 50 \times 10^{9} / \mathrm{L}$ prior to transplantation were assigned a score of one.

The overall score ranged from zero to nine, with increasing scores indicating higher risk of death. Based on the score, four risk groups were delineated: low (0-1), intermediate (2-3), high (4-5), and very high ( $>5)$. The hazard ratio for death (with the low-risk group as the reference) was 2.02 (95\% CI: 1.41-2.90) for the intermediate-risk group, 3.49 (95\% CI: 2.45-4.97) for the high-risk group, and 5.90 (95\% CI: 4.01-8.67) for the very high-risk group. Corresponding survival rates were $68.7 \%$ for the low-risk group, $43.2 \%$ for the intermediate-risk group, $26.6 \%$ for the high-risk group, and $9.5 \%$ for the very high-risk group. Overall, the EBMT transplant-specific risk score was predictive of overall survival $(P<0.001)$ (Figure 1).

\section{Secondary endpoints}

The EBMT cohort was also used to apply the developed score to all secondary objectives. The developed score (overall and in all risk groups) was associated with all secondary endpoints $(P=0.001)$ (Table 3 and Figure 1B-D) The 5-year relapse-free survival rate was $68.4 \%$ (95\% CI: continued from the previous coloum

\begin{tabular}{lc}
$\begin{array}{l}\text { Platelets at transplant, } \mathbf{x} 10^{\circ} \mathrm{L} \\
\leq 50\end{array}$ \\
$>50$ & $382(38)$ \\
\hline Revised IPSS category & $677(62)$ \\
very low & $32(3)$ \\
low & $259(24)$ \\
intermediate & $317(32)$ \\
high & $205(20)$ \\
very high & $86(8)$ \\
unknown & $150(13)$ \\
Conditioning intensity & \\
myeloablative & $336(32)$ \\
reduced & $620(58)$ \\
non-myeloablative & $103(10)$ \\
\hline Pretreatment & \\
chemotherapy & $235(22)$ \\
hypomethylating agents & $214(20)$ \\
both & $42(4)$ \\
none & $568(54)$ \\
Graft source & \\
bone marrow & $147(14)$ \\
peripheral blood & $912(86)$ \\
\hline Time to transplant, months & \\
median (range) & $7(0.2$ to 328$)$ \\
Donor type & \\
HLA-identical sibling & $622(59)$ \\
matched unrelated & $437(41)$ \\
\hline Cytomegalovirus serostatus of recipient & \\
negative & $416(39)$ \\
positive & $614(58)$ \\
unknown & $29(3)$ \\
Anti-thymocyte globulin & $491(46)$ \\
\hline Ex vivo T-cell depletion & $43(4)$ \\
\hline & \\
\hline & \\
\hline & \\
\hline &
\end{tabular}

n: number; IPSS: International Prognosis Scoring System. 
58.4-78.4) for the low-risk group, 43.3\% (95\% CI: 37.249.4) for the intermediate-risk group, $26.0 \%$ (95\% CI: 20.7-31.3) for the high-risk group, and $11.5 \%$ (95\% CI: 5.4-17.6) for the very high-risk group $(P<0.001)$ (Figure $2 \mathrm{~B})$. The cumulative incidence of relapse and non-relapse mortality at 5 years according to risk group were, respectively: $16.9 \%$ and $12.8 \%$ (low-risk group), $28.2 \%$ and $27.7 \%$ (intermediate-risk group), $36.0 \%$ and $36.9 \%$ (highrisk group), and $46.0 \%$ and $44.3 \%$ (very high-risk group) (Figure 2C,D).

\section{Nomogram}

We aimed to refine the transplant-specific risk score further by using age and platelet count at transplantation as continuous variables while the other variables remained in their categorical structure. We provide a discrete/continuous nomogram to interpolate the final score and assess an individual patient's risk in an easy manner (Figure 2). For each of the seven prognostic factors, individual points are assigned, which are subsequently summed to a total point scale. The final score is then translated into predicted survival rates at different time points for each patient. For instance, 55-year old patients presenting with a platelet count of $150 \times 10^{9} / \mathrm{L}$, blasts $\leq 1 \%$, good cytogenetics, a Karnofsky Performance Status of $90 \%$ who are seropositive for CMV and for whom an identical sibling donor is available show estimated survival rates at 3 and 5 years of 57\% (95\% CI: 51-63) and 47\% (95\% CI: 41-54), respectively.

\section{Validation of existing systems}

The overall score from the CIBMTR ranged from zero to seven and was used to define four risk categories: low risk $(0-1, n=46)$, intermediate risk $(2-3, n=434)$, high risk $(4-5, n=365)$, and very high risk $(>5, n=31)$. The 5 -year overall survival rates were $55.8 \%$ (95\% CI: 39.9-71.7) for the low-risk group, $42.3 \%$ (95\% CI: 37.2-47.4) for the intermediate-risk group, and $27.2 \%$ (95\% CI: 21.9-32.5) for the high-risk group. Rates were not estimable in the very high-risk group because the median follow-up was 42.6 months in this group while median overall survival was 11.8 months (95\% CI: 3.1-20.4) (Figure 3A). The GITMO score values ranged from zero to eight. The actual score delineated four risk-groups: low (0-1, $\mathrm{n}=81)$, intermediate (2-3, $\mathrm{n}=200)$, high $(4, \mathrm{n}=123)$, and very high $(>4$, $\mathrm{n}=115)$. The overall survival rates at 5 years were $62.7 \%$ (95\% CI: 51.7-73.7) for the low-risk group, 41.4\% (95\% CI: 34.0-48.8) for the intermediate-risk group, $24.1 \%$ (95\% CI: 15.1-33.1) for the high-risk group, and 15.8\% (95\% CI: 8.2-23.4) for the very high-risk group (Figure 3B). Overall, both scores could be validated $(P<0.001)$.

\section{Comparison of prognostic systems}

Both existing scores from GITMO and CIBMTR were then compared with the developed EBMT transplant-specific risk score with respect to their performance regarding overall survival. The CIBMTR and GITMO scores showed modest performance, with C-statistics after cross-validation being 0.555 (95\% CI: 0.524-0.586) and 0.579 (95\% CI: 0.570-0.588), whereas the IPSS-R resulted in C-statistics of 0.551 (95\% CI: 0.530-0.566). The developed categorized EBMT transplant-specific risk score showed C-statistics of 0.609 (95\% CI: 0.588-0.629) indicating an improvement in prognostic performance, which was further improved using age and platelet count as con-

tinuous variables as indicated by the C-statistics of 0.628 (95\% CI: 0.616-0.637).

\section{Discussion}

The major findings of this analysis can be summarized as follows. First, seven independent risk factors (age, cytogenetics, thrombocytopenia and increasing blood blasts at transplantation, Karnofsky Performance Status, donor relation, and CMV status of the recipient) could be successfully incorporated into a transplant-specific risk score. Second, this EBMT transplant-specific risk score enabled significantly improved prediction of outcome in comparison with currently existing systems.

The EBMT transplant-specific risk score presented here considered conventional clinically derived risk factors at transplantation and can thus be readily calculated. The primary objective in developing this score was to improve our ability to predict survival of MDS patients after transplantation; furthermore, the score was predictive of relapse-free survival, relapse and non-relapse mortality. Moreover, sub-analyses of all MDS patients in the EBMT registry ( $\mathrm{n}=6181$ ), of whom 5122 were not included in the present study because of missing data, revealed a better overall survival rate at 5 years for excluded patients (48\%) compared with that of the patients used to develop the score $(39 \%)$ while non-relapse mortality was $29 \%$, respectively. Most patients had to be excluded because of missing information regarding cytogenetics. Multiple imputation of these patients revealed no difference in score performance. Collectively, the prognostic ability could be improved using the proposed EBMT transplant-specific

Table 2. Multivariate analysis on overall survival showing seven independent risk factors after stepwise selection using a Cox proportional hazards model.

$\begin{array}{lll}\text { Factor } & \text { HR }(95 \% \text { Cl) } & P \quad \begin{array}{l}\text { Score } \\ \text { value }\end{array}\end{array}$

Age at transplant, years

$<50$

$\geq 50$

Blood blasts at transplant, \%

$\leq 1$

$>1$

Platelets at transplant, x10 $/ \mathrm{L}$

$>50$

$\leq 50$

Donor type

HLA-identical sibling

matched unrelated

Cytogenetic risk

very good to poor

very poor/monosomal karyotype

Cytomegalovirus serostatus

of recipient

negative

positive

Karnofsky index, \%

90 to 100

$<90$

HR: hazard ratio; $\mathrm{CI}$ : confidence interval.

$<0.001$

reference

$1.71(1.39-2.09)$

2

reference $1.39(1.03-1.86)$

reference

$1.46(1.17-1.82)$

0.001

1

001


risk score while C-statistics still showed moderate power; thus, prognostic systems in MDS may be validated and updated offering improved risk stratification if more data become available in the registries in the future.

While another study by the EBMT assessed the prognostic utility of the IPSS-R in patients following transplantation, finding modest applicability of this disease-specific approach, ${ }^{17}$ we evaluated two systems that used transplant- and patient-related approaches. The CIBMTR score showed limited to modest performance (0.555) but resulted in an even better prognostic ability (with a concordance index up to 0.582 after cross-validation) in our cohort than originally reported (0.575) while the GITMO score showed better performance (0.579). We acknowledge the limitation of the lack of information regarding the HCT-CI in a sufficient number of patients, which could therefore not be included in the final multivariable model for the development of the EBMT transplant-specific risk score. The Karnofsky Performance Status was used instead. However, the actual performance status of a patient may vary according to clinicians or at different times during the transplantation evaluation. Other tools evaluating patient fitness, including the HCT-CI may be additionally used as they become available in larger populations.

To investigate to what extent a transplant-specific approach will be generally feasible, we evaluated the prognostic power of the IPSS-R resulting in C-statistics of 0.551 (95\% CI: $0.530-0.566$ ), confirming that transplantspecific risk stratification may enable optimized counseling of patients. However, although the proposed EBMT

B
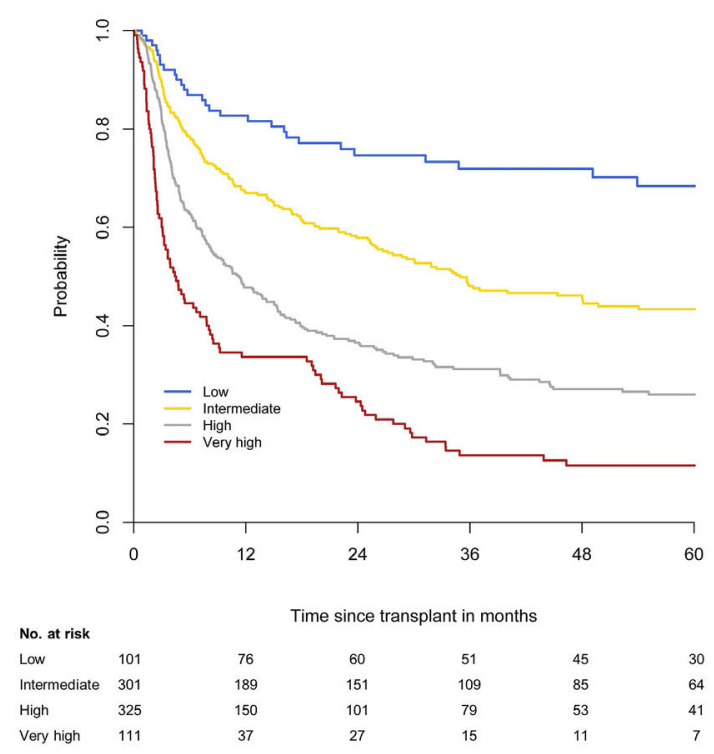

D

EBMT: Non-relapse mortality

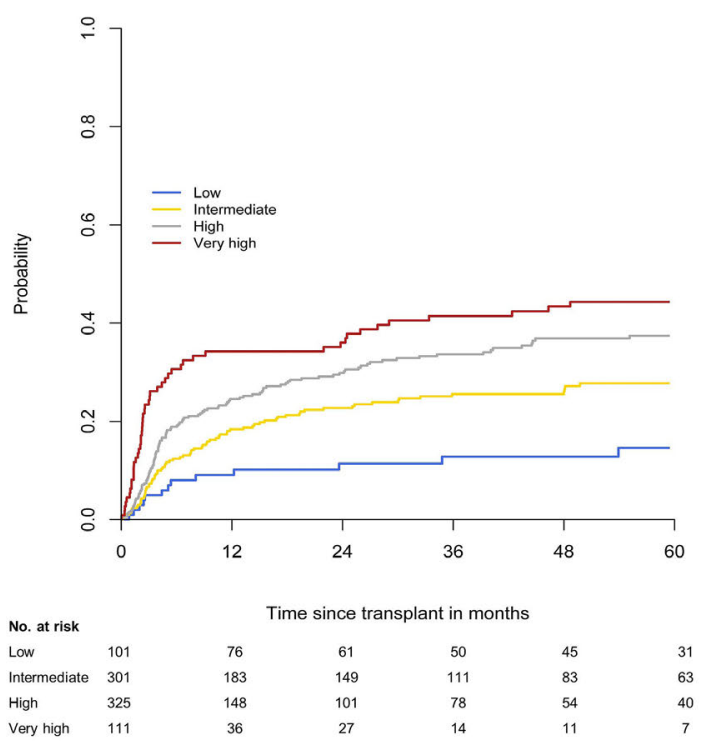


transplant-specific risk score demonstrated improved prognostic capacity over the IPSS-R, the magnitude of this benefit was still moderate, suggesting that the combined use of prognostic systems may provide the most appropriate prognostication, until systems with significantly better performance become available.

None of the existing scores investigated the possible impact of CMV on outcome. CMV is an important cause of morbidity and mortality after allogeneic stem-cell transplantation..$^{18}$ During recent years, major advances have been achieved regarding antiviral prophylactic strategies, and new sensitive diagnostic techniques have been developed. ${ }^{19-21}$ A recent evidence synthesis of the efficacy and safety of different prophylactic strategies for CMV highlighted inconclusive results in terms of survival while CMV disease and infection could be significantly reduced using antiviral agents. ${ }^{20}$ Furthermore, it is unclear whether different prophylactic agents for graftversus-host disease increase the risk for $\mathrm{CMV}$ infection or disease after transplantation. Aggregated evidence did not show an increased risk for CMV reactivation in randomized trials on antithymocyte globulin, which was given to $46 \%$ of our EBMT cohort, in comparison with standard prophylaxis using cyclosporine and methotrex-

Table 3. Transplant-specific MDS risk score prediction of relapse-free survival, non-relapse mortality and incidence of relapse.

\begin{tabular}{|c|c|c|c|c|c|c|}
\hline \multirow[b]{2}{*}{ Risk group } & \multicolumn{2}{|c|}{ Relapse-firee survival } & \multicolumn{2}{|c|}{ Non-relapse mortality } & \multicolumn{2}{|c|}{ Incidence of relapse } \\
\hline & HR (95\% Cl) & $P$ & HR (95\% CI) & $\boldsymbol{P}$ & HR (95\% Cl) & $\boldsymbol{P}$ \\
\hline Score overall & & $<0.001$ & & $<0.001$ & & $<0.001$ \\
\hline low & reference & & reference & & reference & \\
\hline intermediate & $2.03(1.38-2.98)$ & $<0.001$ & $2.08(1.16-3.75)$ & 0.01 & $1.80(1.04-3.10)$ & 0.03 \\
\hline high & $3.47(2.39-5.06)$ & $<0.001$ & $2.99(1.68-5.30)$ & $<0.001$ & $2.68(1.57-4.57)$ & $<0.001$ \\
\hline very high & $5.77(3.85-8.66)$ & $<0.001$ & $4.18(2.25-7.76)$ & $<0.001$ & $3.70(2.09-6.55)$ & $<0.001$ \\
\hline
\end{tabular}

HR: hazard ratio; CI: confidence interval.

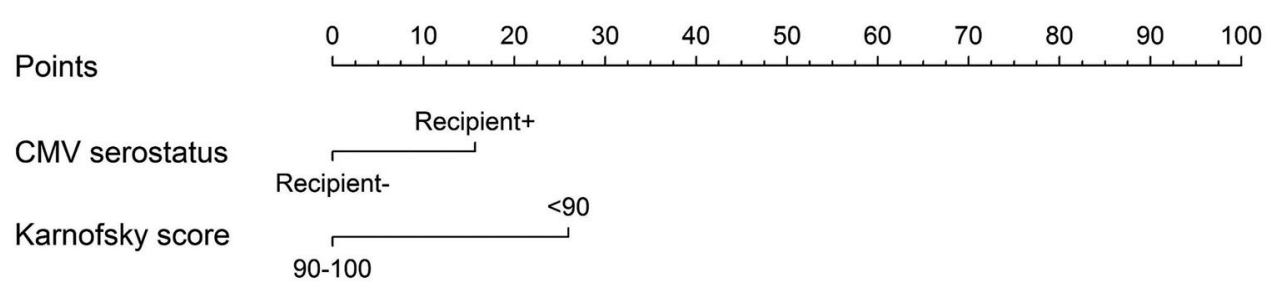
Age at Tx

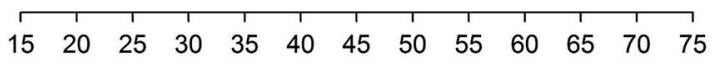

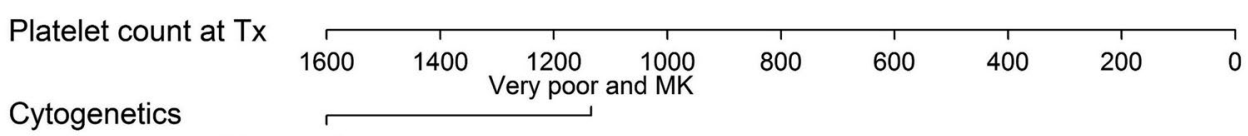

Very good to poor

Donor relation Identical sibling

Total Points

Linear Predictor

$12 \mathrm{~m}$ survival $(\%)$

36 m survival (\%)

60 m survival $(\%)$
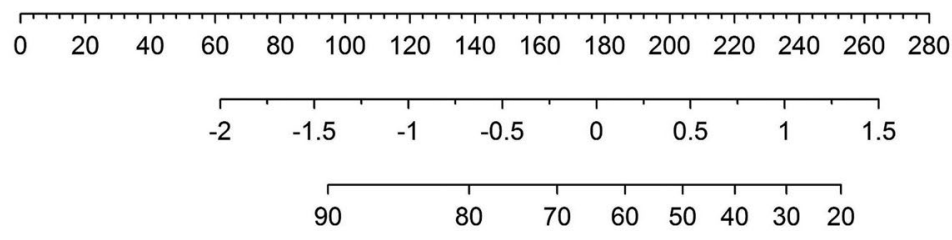

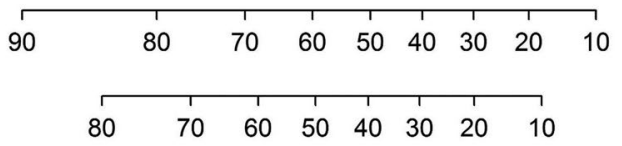

Figure 2. Nomogram of the EBMT transplant-specific risk score: for each of the seven prognostic factors. Corresponding points are assigned, which are subsequently summed to make a total point scale. This final score is then translated into predicted survival rates at different time points for each patient. CMV: cytomegalovirus; Tx: transplantation; MK: monosomal karyotype; 12m: 12-month; 36m: 36-month; 60m: 60-month. 
A

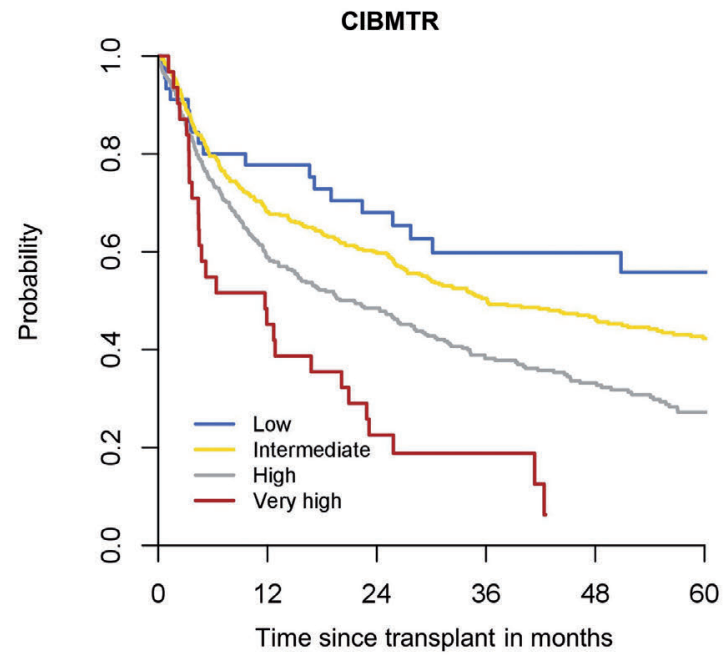

B

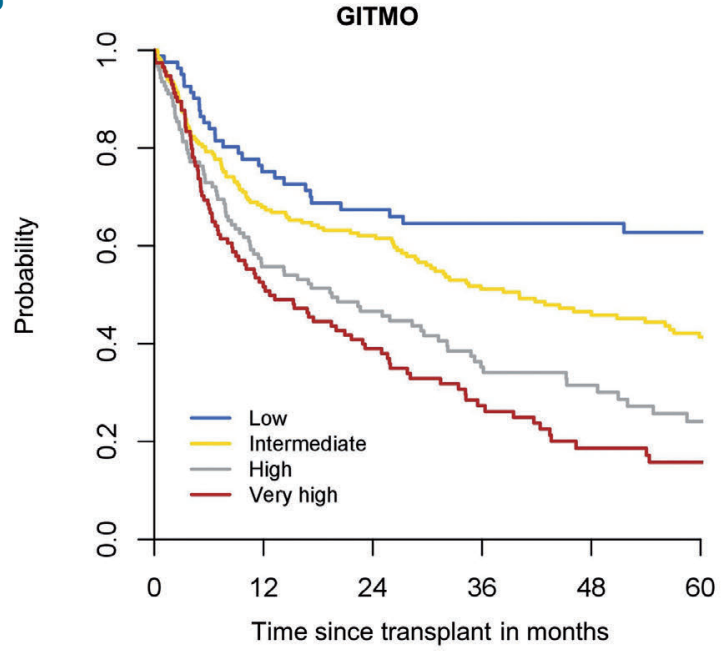

$\begin{array}{lcccccc}\text { No. at risk } & & & & & & \\ \text { Low } & 46 & 33 & 27 & 21 & 16 & 10 \\ \text { Intermediate } & 434 & 284 & 225 & 171 & 137 & 104 \\ \text { High } & 365 & 201 & 151 & 105 & 72 & 49 \\ \text { Very high } & 31 & 14 & 7 & 3 & 0 & 0\end{array}$

$\begin{array}{lcccccc}\text { No. at risk } & & & & & & \\ \text { Low } & 81 & 59 & 49 & 42 & 36 & 30 \\ \text { Intermediate } & 200 & 131 & 110 & 84 & 66 & 52 \\ \text { High } & 123 & 64 & 47 & 32 & 23 & 14 \\ \text { Very high } & 115 & 58 & 41 & 23 & 13 & 11\end{array}$

Figure 3. Kaplan-Meier analysis of survival following allogeneic stem-cell transplantation in patients with myelodysplastic syndrome stratified according to their risk group. (A) Center for International Blood and Marrow Transplant Research (CIBMTR) registry. (B) Gruppo Italiano Trapianto di Midollo Osseo (GITMO) registry.

ate or tacrolimus. ${ }^{22}$ Further analyses, however, showed no significant impact of T-cell depletion or the presence of acute graft-versus-host disease at baseline on outcome of antiviral prophylaxis in terms of CMV infection, survival or safety..$^{20}$ Hence, the CMV serological status of the transplant recipient still has a strong influence on outcome. ${ }^{23}$ In our analysis, a positive CMV serostatus of the recipient was associated with a $39 \%$ increased risk of death, as well as higher rates of non-relapse mortality. The significant impact of CMV on outcome in a multivariable model in this large cohort of MDS patients supports further evaluation of antiviral agents, such as letermovir, affecting not only CMV infection and disease but also mortality. ${ }^{20,21}$

The role of molecular genetics after transplantation has been investigated recently. Most studies ${ }^{24-26}$ found a negative impact on outcome in patients with p53 mutations while one study ${ }^{26}$ suggested that p53 as well as RAS-pathway mutations were mainly seen in patients carrying complex karyotypes. Although molecular genetics have not been included in any existing system and were not available in a suitable number of patients in this analysis, incorporation of genomic aberrations may refine systems in the future.
Regarding conditioning regimens, the introduction of reduced-intensity conditioning has resulted in a significant reduction of transplant-related toxicity and mortality. ${ }^{27}$ Our analysis, in line with the CIBMTR study, found at least similar effects of reduced-intensity and myeloablative regimens. As with any retrospective analysis, these results are prone to bias. Patients perceived as being at greater risk might have been favorably treated using myeloablative conditioning. In our study, patients receiving reducedintensity conditioning were even older and showed a worse performance status than patients given myeloablative conditioning. This observation is supported by a recent prospective study by the EBMT, ${ }^{28}$ in which it was found that the administration of reduced-intensity conditioning before transplantation in $\mathrm{MDS}$ patients resulted in at least an equivalent survival trend for a better overall survival at 2 years, whereas non-relapse mortality appeared to be higher after using myeloablative conditioning.

In conclusion, this EBMT transplant-specific risk score could improve prediction of outcome for MDS patients undergoing allogeneic stem-cell transplantation. This readily available score enables optimized clinical decisionmaking with respect to allogeneic stem-cell transplantation in patients with MDS.

\section{References}

1. Adès $\mathrm{L}$, Itzykson $\mathrm{R}$, Fenaux $\mathrm{P}$. Myelodysplastic syndromes. Lancet. 2014;383(9936):2239-2252.

2. Fenaux P, Mufti GJ, Hellstrom-Lindberg E, et al. Efficacy of azacitidine compared with that of conventional care regimens in the treatment of higher-risk myelodysplastic syndromes: a randomised, open-label, phase
III study. Lancet Oncol. 2009;10(3): 223-232

3. Passweg JR, Baldomero $\mathrm{H}$, Bader $\mathrm{P}$, et al. Hematopoietic stem cell transplantation in Europe 2014: more than 40,000 transplants annually. Bone Marrow Transplant. 2016;51(6):786-792

4. Kröger N. Allogeneic stem cell transplantation for elderly patients with myelodysplastic syndrome. Blood. 2012;119(24):5632 5639.

5. Greenberg P, Cox C, LeBeau MM, et al.
International scoring system for evaluating prognosis in myelodysplastic syndromes. Blood. 1997;89(6):2079-2088.

6. Greenberg PL, Tuechler H, Schanz J, et al. Revised international prognostic scoring system for myelodysplastic syndromes. Blood. 2012;120(12):2454-2465.

7. Kröger N. Maximizing the benefit of allogeneic stem cell transplantation in myelodysplastic syndromes. Semin Hematol. 2017;54 (3):154-158. 
8. Malcovati L, Hellström-Lindberg E, Bowen $\mathrm{D}$, et al. Diagnosis and treatment of primary myelodysplastic syndromes in adults: recommendations from the European LeukemiaNet. Blood. 2013;122(17):29432964.

9. Della Porta MG, Alessandrino EP, Bacigalupo A, et al. Predictive factors for the outcome of allogeneic transplantation in patients with MDS stratified according to the revised IPSS-R. Blood. 2014;123(15): 2333-2342.

10. Shaffer BC, Ahn KW, Hu ZH, et al. Scoring system prognostic of outcome in patients undergoing allogeneic hematopoietic cell transplantation for myelodysplastic syndrome. J Clin Oncol. 2016;34(16):1864-1871.

11. Schanz J, Tüchler H, Solé F, et al. New comprehensive cytogenetic scoring system for primary myelodysplastic syndromes (MDS) and oligoblastic acute myeloid leukemia after MDS derived from an international database merge. J Clin Oncol. 2012;30(17): 820-829.

12. Breems DA, Van Putten WL, De Greef GE, et al. Monosomal karyotype in acute myeloid leukemia: a better indicator of poor prognosis than a complex karyotype. J Clin Oncol. 2008;26(29):4791-4797.

13. Cox DR. Regression models and life tables. J R Stat Soc B. 1972;34:187-220.

14. Fine JP, Gray RJ. A proportional hazards model for the subdistribution of a competing risk. J Am Stat Assoc. 1999;94(9):496509.

15. Gónen M, Heller G. Concordance probability and discriminatory power in proportional hazards regression. Biometrika. 2005;92(4): 965-970.

16. Raykar VC, Steck H, Krishnapuram B,
Dehing-Oberije C, Lambin P. On ranking in survival analysis: bounds on the concordance index. Presented at the 21 Annual Conference on Neural Information Processing Systems, Vancouver, British Columbia, Canada, December 3-6, 2007.

17. Koenecke C, Göhring G, de Wreede LC, et al. Impact of the revised International Prognostic Scoring System, cytogenetics and monosomal karyotype on outcome after allogeneic stem cell transplantation for myelodysplastic syndromes and secondary acute myeloid leukemia evolving from myelodysplastic syndromes: a retrospective multi-center study of the European Society of Blood and Marrow Transplantation. Haematologica. 2015;100(3):400-408

18. Teira P, Battiwalla M, Ramanathan M, et al. Early cytomegalovirus reactivation remains associated with increased transplant-related mortality in the current era: a CIBMTR analysis. Blood. 2016;127(20):2427-2438.

19. Goodrich J, Bowden R, Fisher L, Keller C, Schoch G, Meyers J. Ganciclovir prophylaxis to prevent cytomegalovirus disease after allogeneic marrow transplant. Ann Intern Med. 1993;118(3):173-178.

20. Gagelmann N, Ljungman P, Styczynski J, Kröger N. Comparative efficacy and safety of different antiviral agents for cytomegalovirus prophylaxis in allogeneic hematopoietic cell transplantation: a systematic review and meta-analysis. Biol Blood Marrow Transplant. 2018;24(10): 2101-2109.

21. Marty FM, Ljungman P, Chemaly RF, et al. Letermovir prophylaxis for cytomegalovirus in hematopoietic-cell transplantation. N Engl J Med. 2017;377(25):2433-2444.

22. Gagelmann N, Ayuk F, Wolschke C, Kröger
N. Comparison of different rabbit anti-thymocyte globulin formulations in allogeneic stem cell transplantation: systematic literature review and network meta-analysis. Biol Blood Marrow Transplant. 2017;23(12): 2184-2219.

23. Ljungman P, Brand R, Hoek J, et al. Donor cytomegalovirus status influences the outcome of allogeneic stem cell transplant: a study by the European Group for Blood and Marrow Transplantation. Clin Infect Dis. 2014;59(4):473-481.

24. Della Porta MG, Gallì A, Bacigalupo A, et al. Clinical effects of driver somatic mutations on the outcomes of patients with myelodysplastic syndromes treated with allogeneic hematopoietic stem-cell transplantation. J Clin Oncol. 2016;34(30):3627-3637.

25. Lindsley RC, Saber W, Mar BG, et al Prognostic mutations in myelodysplastic syndrome after stem-cell transplantation. $\mathrm{N}$ Engl J Med. 2017;376(6):536-547.

26. Yoshizato T, Nannya Y, Atsuta Y, et al. Genetic abnormalities in myelodysplasia and secondary acute myeloid leukemia: impact on outcome after stem cell transplantation. Blood. 2017;129(17):2347-2358.

27. Sorror ML, Sandmaier BM, Storer BE, et al. Long-term outcomes among older patients following nonmyeloablative conditioning and allogeneic hematopoietic cell transplantation for advanced hematologic malignancies. JAMA. 2011;306(17):1874-1883.

28. Kröger N, Iacobelli $S$, Franke GN, et al Dose-reduced versus standard conditioning followed by allogeneic stem-cell transplantation for patients with myelodysplastic syndrome: a prospective randomized phase III study of the EBMT (RICMAC trial). J Clin Oncol. 2017;35(19):2157-2164. 